\title{
Bau ermöglicht Entwicklung
}

\section{Einleitung}

$\mathrm{Zu}$ den Leistungen von Hans-Georg Nolte-Fischer als Direktor der Universitäts- und Landesbibliothek Darmstadt gehört es, innerhalb von nur einem halben Jahr zwei Bibliotheksneubauten eröffnet zu haben, wobei er die Bestände der Zentralbibliothek mit denen von über zwanzig dezentralen Bibliotheken vereinigte, die aus der vormals großen Zahl der nach dem Kriege entstandenen Instituts-, Arbeitsgruppen- und Fachbereichsbibliotheken verblieben waren. Mit der Konzentration der zuvor stark zergliederten Bibliothekslandschaft, der Erhöhung der Zahl der Arbeitsplätze und dem Ausbau des Freihandangebots war es in Darmstadt gelungen, die Bibliothek strikt auf die Nutzerbedürfnisse hin auszurichten; dies spiegelt zugleich eine paradigmatische Entwicklung für alle zweischichtigen Bibliothekssysteme wider ${ }^{1}$.

Auch wenn die Universitätsbibliothek der LMU München bisher noch auf keinen zentralen Bibliotheksneubau zurückblicken kann, erscheinen die Ausgangssituation, aber auch der eingeschlagene Weg in München wie in Darmstadt in vielem ähnlich und vergleichbar, so dass es lohnend erscheint, hier den Münchner Weg näher zu beleuchten².

Dabei zeigt sich, dass, anders als in der gängigen Literatur zum Bibliothekbau idealtypisch beschrieben ${ }^{3}$, die bauliche Entwicklung eines Bibliothekssystems nicht autonom nach hehren Grundsätzen geplant werden kann, sondern auch auf günstige Zeitfenster und Konstellationen angewiesen ist.

\section{Die Ausgangssituation}

Die Universitätsbibliothek München war vor dem Zweiten Weltkrieg die größte deutsche Universitätsbibliothek. In den Bombennächten der Jahre 1943/44, insbesondere bei einem Großangriff auf München am 13. Juli 1944, wurden die Bibliothek und die gesamten Universitätsgebäude vollständig zerstört. Die Weiterexistenz der Universitätsbibliothek war

$1 \mathrm{Vgl}$. Nolte-Fischer, Hans-Georg: Die Bibliothek. Funktion - Architektur - Organisation. In: Neue Mitte[n]. Die Bibliotheksneubauten der Technischen Universität Darmstadt, hrsg. von der Universitätsund Landesbibliothek Darmstadt und der Technischen Universität Darmstadt. Darmstadt 2014, S. 117 124, hier S. 117-118; sowie Nolte-Fischer, Hans-Georg: Funktionale Einschichtigkeit: von der gesetzlichen Normienung zur praktischen Umsetzung - das Beispiel Darmstadt. In: Zeitschrift für Bibliothekswesen und Bibliographie 49 (2002), S. 283-288.

2 Teile dieses Aufsatzes gehen auf einen vom Verfasser am 16.03.2016 beim 6. Bibliothekskongress in Leipzig u. d. T. ,Bibliotheksbau und Reorganisation an der UB der LMU* gehaltenen Vortrag zurück.

3 So beispielsweise Naumann, Ulrich: Grundsätze des Bibliotheksbaus - Von den ,Zehn Geboten' von Harry Faulkner-Brown zu den ,Top Ten Qualities‘ von Andrew McDonald. In: Bibliotheken bauen und ausstatten, hrsg. von Petra Hauke und Klaus Ulrich Werner. Bad Honnef 2009, S. 14-37. - Vgl. weiterhin Klaus Ulrich Werner und Jonas Fansa: Change Management - Potenziale und Risiken des Bibliotheksbauprozesses. In: Bibliotheken bauen und ausstatten (wie oben), S. 132-136. 
fraglich; zwar war der Altbestand ganz überwiegend ausgelagert und gerettet worden, doch der übrige Bestand und ein Teil der Kataloge waren verbrannt, Lesesäle und Arbeitszimmer waren unter Trümmern begraben. Der naheliegende, unmittelbar nach Kriegsende entwickelte Gedanke, die Universitätsbibliothek unter dem organisatorischen Dach der ebenfalls schwer beschädigten und räumlich benachbarten Bayerischen Staatsbibliothek wieder aufzubauen, kam über eine erste frühe Phase nicht hinaus. $\mathrm{Zu}$ unvorstellbar war es für die Universität, auf eine eigene Bibliothek zu verzichten, und ebenso unvorstellbar für die Staatsbibliothek, ihre Selbständigkeit aufzugeben und Teil einer Universität zu werden ${ }^{4}$.

Doch mit der Aufgabe der Vereinigungspläne stockte auch der Wiederaufbau der Universitätsbibliothek. Während die Universitätsgebäude nach und nach wieder aufgebaut wurden, hauste die Universitätsbibliothek mehr als 20 Jahre lang in Ruinen und Provisorien. Erst 1967, nach einem öffentlichen Skandal um den verrottenden Altbestand, der sich immer noch in Teilen in für eine dauerhafte Lagerung völlig ungeeigneten Auslagerungsstätten befand, wurden für die Universitätsbibliothek im Südflügel des Hauptgebäudes wieder eigene Räumlichkeiten errichtet, deren Kernstück ein großes Magazin hinter der rekonstruierten historischen Front zur Ludwigstraße bildete 5

In den 22 Jahren nach Kriegsende, in denen die Universitätsbibliothek nahezu unsichtbar bleiben musste, ging die Literaturversorgung für die Wissenschaftler und Studierenden der größten Universität Deutschlands weitgehend auf bereits bestehende oder neu errichtete Instituts-, Seminar- und Lehrstuhlbibliotheken über; selbst das Studentenwerk unterhielt als bis in die jüngste Vergangenheit bestehende Besonderheit - eine eigene Bibliothek mit einem großen Angebot an Lehrbüchern und Freizeitliteratur. Dieser Zustand hielt auch lange nach dem Wiederaufbau der Universitätsbibliothek noch an: Wissenschaftler und Studierende nutzten überwiegend die Institutsbibliotheken, die Wissenschaftler zudem die damals für Studierende weitgehend unzugängliche Staatsbibliothek. Der Universitätsbibliothek verblieb ein Nischendasein, das sich im Wesentlichen auf die Pflege des Altbestandes und eines aufwändigen systematischen Katalogs, auf die Bearbeitung des aus dem Regierungsbezirk Oberbayern stammenden Pflichtzugangs sowie auf die Erwerbung eines kleinen Grundbestandes ausleihbarer Literatur beschränkte.

Sejt den 1970er Jahren bemühte sich die Universitätsbibliothek um eine verstärkte Koordination der formal völlig selbständigen und den einzelnen Instituten, Seminaren oder Kliniken zugeordneten Bibliotheken, die zudem über das ganze Stadtgebiet von München verteilt und nicht selten in den ehemaligen Wohnzimmern früherer Wohngebäude eingerichtet waren. Die Universitätsbibliothek gründete dafür eine sogenannte Kontaktstelle; die Mitarbeiter der Kontaktstelle übernahmen neben koordinierenden Aufgaben vielfach die Formalerschließung an den kleineren Bibliotheken und schulten das Personal der etwas größeren Institutsbibliotheken.

$4 \mathrm{Vgl}$. Kuttner, Sven: „Die größte Sorge der Bibliothek aber ist die furchtbare Raumnot“. Die Gebăudeentwicklung der Universitätsbibliothek München im 19. und 20. Jahrhundert. In: Bibliotheksdienst 45 (2011), S. 442-452. - Vgl. weiterhin Kuttner, Sven: Vom Prachtbau zur Kompromisslösung. Die bauliche Entwicklung der Münchner Universitätsbibliothek im 19. und 20. Jahrhundert. In: Domus universitatis. Das Hauptgebäude der Ludwig-Maximilians-Universität 1835-1911-2011, hrsg. von Claudius Stein. München 2015, S. 111-122.

$5 \mathrm{Vgl}$. die beiden in Anm. 4 genannten Beiträge von Kuttner. - Siehe weiterhin auch: Buzás, Ladislaus: Geschichte der Universitätsbibliothek München. Wiesbaden 1972, S. 233 und S. 248-255. 


\section{Der Aufbau eines einheitlichen Bibliothekssystems - 30 Jahre Fachbibliotheken an der LMU}

Eine grundlegende Wende in der Rolle der Universitätsbibliothek für die Literaturversorgung der Universität und ihrer Fakultäten ergab sich mit der Planung der ersten Fakultätsbibliothek Mitte der 1980er Jahre. Damals wurde für die aus einer früher selbstständigen Pädagogischen Hochschule hervorgegangenen und noch lange im entfernten Stadtteil Pasing untergebrachten Erziehungswissenschaften sowie für das damals recht junge Fach Psychologie ein neues Gebäude errichtet, einige hundert Meter vom Hauptgebäude entfernt. Erstmals - und dies stellte sich später als die entscheidende Weichenstellung heraus - war nicht nur beschlossen worden, für diese beiden Fächer eine gemeinsame Fakultätsbibliothek einzurichten, sondern diese zugleich organisatorisch mit der Universitätsbibliothek zu verbinden. Zum Wintersemester 1985/86 wurde die Bibliothek eröffnet und ihre Leitung einer Bibliothekarin des höheren Dienstes der Universitätsbibliothek übertragen ${ }^{6}$.

Die Eröffnung dieser Bibliothek war der Beginn eines Bruchs mit der bisherigen absoluten Vorherrschaft der Institutsbibliotheken. Es brauchte jedoch noch bis zum Ende der 1990er Jahre, bis sich die Konturen eines einheitlichen Bibliothekssystems erkennen ließen, das aus einer Zentralbibliothek und in der Regel auf Fakultätsebene organisierten Fachbibliotheken besteht. Dass dieser ersten organisatorischen Veränderung in Richtung eines funktional-einschichtigen Bibliothekssystems zunächst eine Baumaßnahme zugrunde lag, bildete so etwas wie eine Blaupause für alle künftigen organisatorischen Veränderungen im Bibliothekssystem der LMU. Meist waren es weniger bibliothekarische Konzepte oder Überlegungen, die den Anstoß und die Durchsetzungskraft für Veränderungen gaben, sondern liegenschaftliche Bedürfnisse und bauliche Notwendigkeiten. Dazu muss man wissen, dass der ursprüngliche Campus der LMU am Rand der Münchner Altstadt, direkt an der in der Mitte des 19. Jahrhunderts als Prachtstraße gebauten Ludwigstraße liegt, am Übergang nach Schwabing - mithin in einer der teuersten und attraktivsten Gegenden der Stadt, in der auch Großkonzerne wie die Münchner Rück oder die Allianz ihren Sitz in unmittelbarer Nachbarschaft haben. Raum ist daher die knappste Ressource der LMU, und angesichts des Platzmangels waren und sind auch noch viele Einrichtungen der LMU in andere Stadtteile, ja bis über die Stadtgrenze hinaus ausgelagert und dabei nicht selten in für Forschung und Lehre ungeeigneten Gebäuden untergebracht. Auch wenn die baulichen Veränderungen selten von der Bibliothek selbst ausgehen konnten, sondern den geschilderten Notwendigkeiten folgen mussten, ist es der Universitätsbibliothek gelungen, in enger und erfolgreicher Zusammenarbeit mit dem Dezernat Liegenschaften der Universitätsverwaltung und dem Staatlichen Bauamt, das Bibliothekssystem in einem Zeitraum von zwei Jahrzehnten mit Hilfe einer größeren Zahl von Neu- und Umbauten auf eine völlig neue organisatorische Basis zu stellen ${ }^{7}$.

6 Vgl. Ostmann, Torsten: 30 Jahre Fachbibliotheken an der LMU München. Der Weg zu einem modernen und leistungsfähigen Bibliothekssystem. In: Bibliotheksforum Bayern 10 (2016), S. 40-41; sowie: Janello, Christoph: Der Weg zur optimal strukturierten Informationsversorgung an Universitäten. In: b.i.t. online 18 (2015), S. 564-565.

$7 \mathrm{Vgl}$. Söllner, Konstanze: Bauliche Rahmenbedingungen bei der Zusammenlegung von Institutsbibliotheken. Die Umnutzung eines Flügels des historischen Universitäts-Hauptgebäudes für die ,Bibliothek Theologie - Philosophie'. In: ABl-Technik 25 (2005), S. 260-265, hier S. 263. 
Abgesehen von einigen kleineren Bibliothekszusammenlegungen machte der Bau des 1999 eröffneten Historicums den Anfang. Im Bereich der Geschichtswissenschaft war die Zersplitterung besonders eklatant. Die einzelnen historischen Lehrstühle waren über mehrere Münchner Stadtteile verteilt und mitsamt der jeweiligen Institutsbibliothek überwiegend in früheren Wohngebäuden untergebracht. Das neue Konzept sah vor, alle historischen Lehrstühle - sowie aus Platzgründen noch Lehrstühle einiger mehr oder weniger naheliegender Fächer wie der Ur- und Frühgeschichte oder der Byzantinistik - in einem Gebäude in unmittelbarer Nähe des Universitätshauptgebäudes unterzubringen, in dessen Zentrum eine gemeinsame und einheitliche Bibliothek geplant wurde. Der vom Staatlichen Bauamt gefertigte Entwurf, der einen zuvor von der (zwischenzeitlich der Technischen Universität München zugeordneten) Forstwissenschaftlichen Fakultät genutzten Altbau mit einem neuen, sehr lichten Bibliotheksbau verband, entfaltete für die weitere Entwicklung des Bibliothekssystems eine geradezu paradigmatische Wirkung: Bei den künftigen Neuund Umbauplanungen wurde stets die Bibliothek mit den Räumlichkeiten für Lehrstühle und Institute sowie den Hörsälen und Seminarräumen baulich verbunden; in den Geisteswissenschaften bildet die Bibliothek auch meist zugleich das Zentrum des neuen Gebäudes. Noch beim Neubau des Gebäudes für die Psychologie und Pädagogik war die Bibliothek jedoch im Gesamtgebäude von außen kaum wahrzunehmen. Davon hob sich der Entwurf des Staatlichen Bauamts für das Historicum deutlich ab und unterstrich die zentrale Positionierung der Bibliothek sehr eindrucksvoll, was sich besonders deutlich am Abend zeigt, wenn die Glasfassade der Bibliothek hell erleuchtet in den vom Universitätshauptgebäude gesäumten Innenhof strahlt und den Blick auf Bücherregale und die in der Bibliothek arbeitenden Studierenden freigibt.

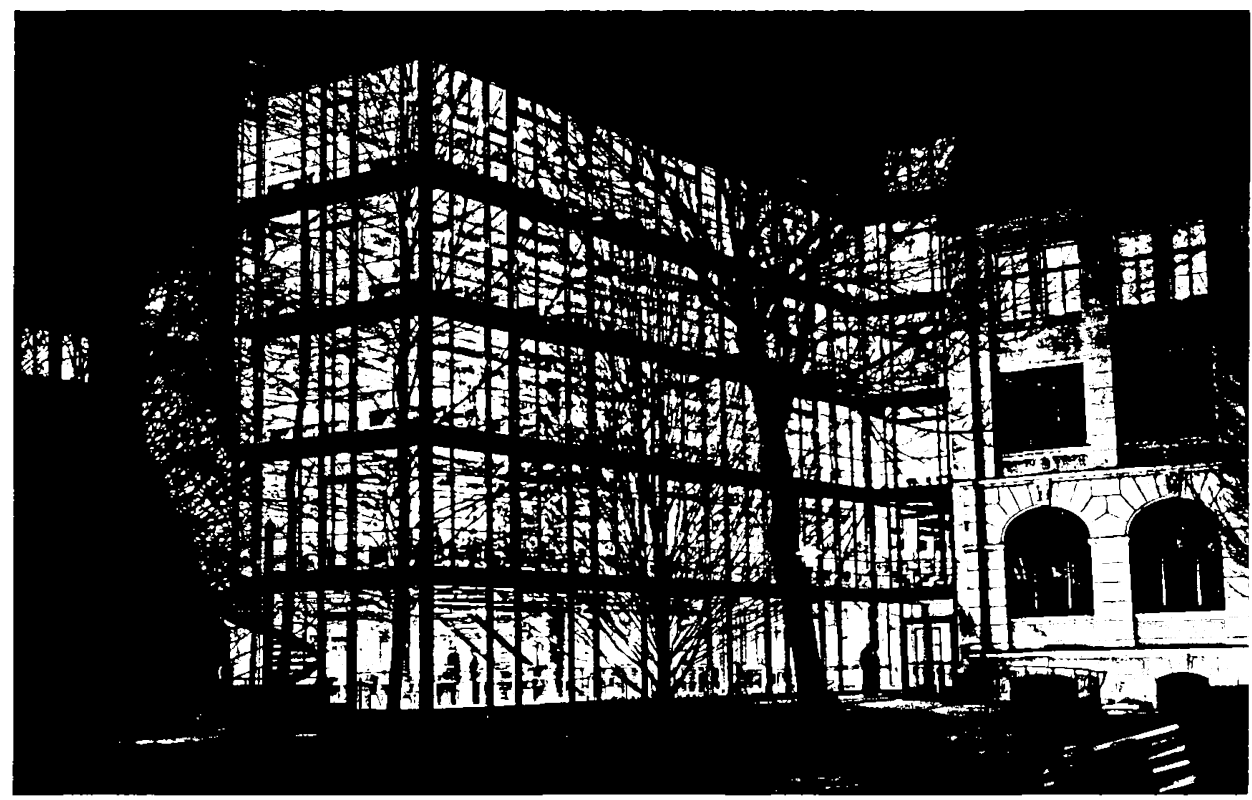

Abb. 1: Auch bei Nacht ein Blickfang. Die beleuchtete Fachbibliothek Historicum.

Foto: Benjamin Rücker 
Dass sich in der Folgezeit die auf das gesamte Gebäude bezogene Bezeichnung Historicum auch als Synonym für die darin gelegene Fachbibliothek eingebürgert hat, zeigt ebenfalls die zentrale Bedeutung der Bibliothek. Natürlich war die Zusammenlegung der Institutsbibliotheken zu einer gemeinsamen Fachbibliothek ebenso wenig konfliktfrei verlaufen wie die gesamte räumliche Zentralisierung der verteilten Institute, und es bedurfte erheblicher Anstrengungen, um die zentrifugalen Kräfte in der Fakultät einzugrenzen. Das enge Zusammenwirken von Universitätsverwaltung und Bibljothek, aber auch der Druck der knappen Räumlichkeiten waren Faktoren, die das Projekt letztlich zum Erfolg werden ließen. Dass ein Institut nicht in das gemeinsame Gebäude eingezogen ist und auch weiterhin eine eigene Institutsbibliothek unterhält, zählt zu den Kompromissen, die hingenommen werden mussten.

Der Erfolg beim Bau des Historicums, bei dem es überdies gelang, die geplante Bausumme zu unterschreiten ${ }^{8}$, war ein wesentlicher Impuls für die Planung einer weiteren, 2004 fertiggestellten, geisteswissenschaftlichen Bibliothek. Im renovierungsbedürftigen Nordflügel des Universitätshauptgebäudes wurde ein bisher als Bürotrakt genutzter Bereich zu einer neuen Fachbibliothek mit einem Fassungsvermögen von rund 350.000 Bänden umgebaut, um die bis dahin auf 17 Standorte verteilten Bestände der beiden Theologischen Fakultäten und der Philosophie aufzunehmen. Unter dem Arbeitstitel ,Bücherturm entstand dabei eine Bibliothek, die sich um ein zentrales Treppenhaus gruppiert und auf fünf Stockwerken rund 350.000 Bände aufnehmen konnte. Die Umbauten für die theologischen und philosophischen Lehrstühle folgten in diesem Fall erst mit einiger zeitlicher Verzögerung, so dass die Fachbibliothek Theologie-Philosophie bei der räumlichen Integration des Theologicums die Vorreiterrolle innehatte'. Bei der Fertigstellung der Fachbibliothek Theologie-Philosophie waren die strukturbildenden Maßnahmen der Universitätsbibliothek bereits so weit fortgeschritten, dass die Bibliothek von vornherein als Fachbibliothek der Universitätsbibliothek geplant wurde - mit nur einem Eingang, während beim Historicum noch auf jedem Stockwerk Seiteneingänge für den Lehrkörper vorgesehen werden mussten. Auch die unter Konfessionsgesichtspunkten nicht ganz unkritische Frage der Durchmischung katholischer und evangelischer Bestände wurde zumindest im Ergebnis nicht in Frage gestellt. Lediglich die orthodoxe Theologie - eine Besonderheit in München - wurde nicht integriert und unterhält nach wie vor eigene Bibliotheksräumlichkeiten.

Für die Naturwissenschaften war die Zeit zwischen der Eröffnung des Historicums und dier Bibliothek Theologie-Philosophie durch den sukzessiven Umzug auf den Campus Großhadern im Süden der Stadt bzw. den angrenzenden, bereits außerhalb der Münchner Gemarkung liegenden Campus Martinsried geprägt. Mit dem 1999 erfolgten Umzug der Chemie und Pharmazie in ein neues Lehr- und Forschungsgebäude wurde darin auch die knapp 80.000 Bände und 143 Arbeitsplätze umfassende Fachbibliothek Chemie und Pharmazie eingerichtet. 2008 folgte die Gründung der Fachbibliothek Biologie - bis dahin existijerten nur einzelne Institutsbibliotheken - auf dem damals neuen Campus Martinsried ${ }^{10}$.

8 Ebd., S. 262.
9 Ebd., S. 265.

$110 \mathrm{Vgl}$. dazu Trapp, Nikola: Die Universitätsbibliothek München fusioniert ihre biowissenschaftlichen Institutsbibliotheken: Zur Neueröffnung der Bibliothek des Biozentrums. In: Bibliotheksdienst 42 (2008), S. 1172-1178. 
Aufgrund der schlechten Verkehrserschließung dieses Campus und der im Vergleich zu den Geisteswissenschaften eher untergeordneten Bedeutung der Bibliothek im klassischen Sinne erschienen die geplanten Dimensionen der Fachbibliothek im Biozentrum mit nur achtzig Arbeitsplätzen zunächst ausreichend. Mit der Entscheidung der Universität, auf dem Campus Martinsried nicht nur ein neues Biomedizinisches Centrum (BMC) als großes Forschungsgebäude einzurichten, sondern auch die vorklinische Lehre weitgehend nach Martinsried zu verlagern, erwies sich alle bisherige Infrastruktur dieses Standortes als unzlreichend - dies betrifft neben der Mensa und der immer noch unbefriedigenden Verkehrserschließung auch die Größe der Bibliothek. Zwar gelang es, die für das BMC geplante kleine Bibliothek kurz vor Eröffnung organisatorisch mit der Fachbibliothek Biologie zu einer neuen Fachbibliothek Biologie und Biomedizin zusammenzuschließen. Die Aufteilung auf zwei gegenüberliegende Gebäude ist jedoch misslich, trotz des aus der Not geborenen, aber gut vermittelbaren Konzeptes, in dem einen Gebäude die Lehrbuchsammlung für beide Fächer und in dem anderen die Präsenzliteratur und den größten Teil der Arbeitsplätze unterzubringen. Hier zeigen sich die Grenzen des sehr pragmatischen Vorgehens an der LMU, bei der die liegenschaftlichen Planungen stets in Kategorien wie Fakultäten oder Fächern erfolgen, aber die übergreifenden Strukturen, die bei bibliothekarischen Konzeptionen tragend sind, ebenso wie langfristige Aspekte leicht in den Hintergrund geraten.

\section{Die Weiterentwicklung seit 2011}

Mit dem Abschluss dieser Baumaßnahmen umfasste die Universitätsbibliothek zehn Fachbibliotheken und eine Zentralbibliothek, die seit 2011 auf zwei Standorte verteilt ist - neben der eigentlichen Zentralbibliothek noch die mehrere Straßenblöcke entfernt liegende Zentrale Lehrbuchsammlung, die auf die oben schon erwähnte ehemalige Studentenbibliothek des Studentenwerks zurückgeht, die 2010 mit veränderter Konzeption von der UB übernommen wurde". Seit 2007 sind das Zusammenspiel von Zentral- und Fachbibliotheken und die Entwicklung zur funktionalen Einschichtigkeit auch in der Grundordnung der LMU festgehalten ${ }^{12}$. Am Ende des ersten Jahrzehnts dieses Jahrhunderts deckten die Fachbibliotheken der Universitätsbibliothek fast das gesamte Fächerspektrum der LMU ab, jedoch fehlten insbesondere in den Rechtswissenschaften, in den weitgespannten Kulturwissenschaften, in den Geowissenschaften und in den Philologien eigene Fachbibliotheken. Während in den Rechtswissenschaften ein nach wie vor dominierendes Institutsdenken alle Pläne für eine einheitliche Fachbibliothek bereits im Ansatz hat ersticken lassen, erschien es bei der großen Heterogenität der kulturwissenschaftlichen Fächer schwierig und wohl auch nicht ratsam, diese in einer Fachbibliothek zu vereinen. Gleichwohl betreuen einige Fachbibliotheken einzelne Fächer der Kulturwissenschaften; überwiegend unterhalten die kulturwissenschaftlichen Fächer jedoch eigene Institutsbibliotheken.

11 Vgl. Heischmann, Günter; Kuttner, Sven; Söllner, Konstanze: Neue Wege in der studentischen Literatur- und Informationsversorgung: Die Studentenbibliothek wird eine Teilbibliothek der Universitätsbibliothek München. In: Bibliotheksdienst 42 (2008), S. 542-549.

12 Dort heißt es: Die Universitätsbibliothek ,umfasst den gesamten Bücherbestand der Universität und gliedert sich in die zentrale Bibliothek und in Fachbibliotheken" ( $\$ 4$ der Grundordnung der LudwigMaximilians-Universität München vom 15. Juni 2007). 
In den letzten drei bis vier Jahren hat sich dagegen sowohl für die Geowissenschaften als auch für die Philologien jeweils eine bauliche Lösung aufgezeigt, die zusammen mit der grundlegenden Sanierung der sogenannten Medizinischen Lesehalle die größten Bau- und Organisationsprojekte der UB der LMU der letzten Jahre bildeten.

Dabei war die im Herbst 2015 eröffnete Fachbibliothek Geowissenschaften das überraschendste Projekt, bei dem sich wiederum zeigte, dass einerseits die bibliotheksorganisatorische Entwicklung ganz wesentlich von baulichen Veränderungen vorangetrieben werden kann, während gleichzeitig diese baulichen Veränderungen liegenschaftlichen Notwendigkeiten folgen, wobei die dauerhafte, intensive Zusammenarbeit zwischen dem Liegenschaftsreferat der Universität und der Universitätsbibliothek die Basis bildet, um in günstigen Momenten langfristig angedachte Planungen zu verwirklichen. So war auch die Bildung einer einheitlichen Fachbibliothek Geowissenschaften, zu der die drei - schon lange durch Personal der Universitätsbibliothek betreuten - Institutsbibliotheken für Geowissenschaften, Geographie sowie Wirtschafts- und Sozialgeographie (eine vierte Bibliothek war kurz zuvor bereits durch die Universitätsbibliothek aufgelöst worden) vereint werden sollten, schon längere Zeit zwischen Universitätsbibliothek und Liegenschaftsreferat erörtert worden. Die Vielzahl der an der LMU anstehenden dringenden Bauprojekte ließ jedoch eine Umsetzung bestenfalls in ferner Zukunft realistisch erscheinen. Jedoch ergab sich ein günstiger Moment, als die Institutsbibliothek für Wirtschafts- und Sozialgeographie, die nach der Neuzuordnung von der TU zur LMU infolge der in den 1990er Jahren erfolgten Neustrukturierung der beiden Münchner Universitäten noch in einem Gebäude der TU verblieben war, dort ausziehen musste. Durch den engen Kontakt zwischen Universitätsbibliothek und dem Liegenschaftsreferat ergab sich die Chance, anstelle einer bloßen Bibliotheksverlagerung eine zusammenhängende und einheitliche Fachbibliothek einzurichten. Planmäßige Haushaltsmittel standen dafür freilich nicht zur Verfügung. Die gesamte Baumaßnahme musste aus dem Bauunterhalt finanziert werden, was auf der einen Seite ein erhebliches Entgegenkommen des Liegenschaftsreferats bedeutete, auf der anderen Seite der Maßnahme ein von vornherein enges finanzielles Korsett auferlegte.

Die neue Fachbibliothek wurde in einer vertikalen Flucht des Bestandsgebäudes geplant - sie erstreckt sich über alle Stockwerke des Gebäudes und wird durch ein bereits vor Beginn der Baumaßnahme bestehendes zentrales Treppenhaus erschlossen. Für den Umbau mussten nicht nur die horizontalen Erschließungswege des Gebäudes gegenüber der Bibliothek abgeschottet werden, es wurde auch die bestehende kleinräumige Struktur zugunsten großzügiger Bibliotheksräume aufgebrochen; zugleich wurden zusätzliche Gruppenarbeitsräume eingebaut. Architektonischer Höhepunkt des Gebäudes ist das ,Penthouse - ein Lernbereich im obersten Stockwerk mit Blick über die gesamte Stadt bis hin zu den Alpen. Für die bibliothekarische Nutzung dieses vormals als Stellplatz für Zeichengeräte und als Teeküche der Institute unterwertig genutzten Raumes musste, um den Brandschutzanforderungen Genüge zu tun, eigens ein zusätzliches Treppenhaus eingebaut werden. Mit 248 Arbeitsplätzen und Stellplatz für rund 90.000 Bände wurde das Verhältnis von Stellplatz und Arbeitsplätzen gegenüber den vorangegangenen, von der Menge der unterzubringenden Bände dominierten Projekte umgekehrt und entspricht damit auch den modernen bibliothekarischen Anforderungen. 
Die Umsetzung zeitgemäßer bibliothekarischer Konzepte gelang auch bei dem grundlegenden Umbau der sogenannten Medizinischen Lesehalle in den Jahren 2011-2013. In Gegensatz zu den vorangegangenen Projekten ging es dabei nicht um die Neueinrichtung einer Fachbibliothek, sondern um die Sanierung einer bestehenden Bibliothek. Auch hir sichtlich ihrer Entstehungsgeschichte weist die im Klinikviertel liegende Medizinische Lesehalle eine Besonderheit auf: Sie war niemals Teil eines Institutes oder einer anderen wissenschaftlichen Einrichtung, sondern wurde 1930 als medizinische Außenstelle der Universitätsbibliothek eröffnet. Dazu hatte eine deutsch-amerikanische Ärztin und Mäze natin das zu Beginn des Jahrhunderts von einem bekannten Münchner Architekten im Jugendstil als Galerie errichtete Gebäude für die Universität erworben. Im Krieg wurde diese Außenstelle der Universitätsbibliothek, ganz im Gegensatz zur Hauptbibliothek, nur geringfügig beschädigt, aber daher auch nur in sehr geringem Umfang renoviert ${ }^{13}$. Trotz einer teilweisen Neumöblierung und kleineren ,Aufpoliermaßnahmen' wirkte die Bibliothek altmodisch, verwinkelt und leicht heruntergekommen; die Jugendstilmerkmale des Gebätr des waren z. T. durch Einbauten verdeckt und fielen kaum mehr ins Auge. Auch wenn der Anlass für diese Baumaßnahme zunächst bauliche und liegenschaftliche Notwendigkeiten waren, so ließen sich im Zusammenspiel von Universitätsbibliothek, LMU-Liegenschaftsreferat und staatlichem Bauamt grundlegende bibliothekarische Ziele umsetzen. Aus baulicher Sicht hatte die Renovierung das Ziel, den Brandschutz zu ertüchtigen, einen barrierefreien Zugang zu gewährleisten und die sanitären Anlagen zu sanieren, was aus hygienischen Gründen dringend erforderlich war. Diese notwendigen Sanierungen und Umbauten vor Augen hatte die Universitätsbibliothek die Gelegenheit genutzt, um die bibliothekarische Konzeption der Medizinischen Lesehalle grundlegend zu überdenken und mit der Neuplanung den heutigen funktionalen Anforderungen an Bibliotheksgebäude gerecht zu werden.

Dabei ging es zunächst darum, das ursprünglich sehr bestandsdominierte und vom Gedanken einer umfassenden medizinischen Zentralbibliothek geprägte Konzept der Medizinischen Lesehalle, in der ein großer Teil der verfügbaren Fläche für die Auslage gedruckter Zeitschriftenhefte und die Aufstellung gebundener Zeitschriftenbände sowie eines größeren Monographienbestandes verwendet worden war, in ein stärker nutzungsorientiertes Konzept zu überführen. Das im Keller der Medizinischen Lesehalle bestehende umfangreiche Magazin wurde grundsätzlich beibehalten, jedoch von Dubletten und entbehrlichen Beständen bereinigt; die dabei freigewordenen Flächen konnten - sofern sie nicht für technisch erforderliche Installationen benötigt wurden - einen großen Teil der bisher in den oberen Stockwerken untergebrachten und für die tägliche Nutzung nicht erforderlichen Bestände aufnehmen. Zugleich hatte die Universitätsbibliothek entschieden, für den bisher schon in der Medizinischen Lesehalle untergebrachten Teil der Lehrbuchsammlung künftig Selbstausleihe mit RFID anzubieten. Damit konnten wenig ästhetische und dem Brandschutz zuwiderlaufende Einbauten wie Drehkreuze und Absperrschranken entfallen.

$13 \mathrm{Vgl}$. Schüller-Zwierlein, André: How Buildings Learn. Zwei Jahre neue Medizinische Lesehalle. In: Bibliotheksforum Bayern 9 (2015), S. 284-288. - Weiterhin: Buzas: Geschichte (wie Anm. 5), S. 225227. 
Vor der grundlegenden Umgestaltung stand jedoch der vorübergehende Auszug der gesamten Bibliothek. Es war ein glücklicher Zufall, dass das ehemalige Mutterhaus des vormals in der Medizinischen Universitätsklinik ansässigen Schwesternordens vorübergehend leer stand und die Medizinische Lesehalle in der profanisierten Hauskapelle des früheren Mutterhauses ein provisorisches, aber zugleich außergewöhnliches Ausweichquartier finden konnte.

Bei der Neugestaltung der Medizinischen Lesehalle wurde großer Wert auf eine hohe Aufenthaltsqualität in der Bibliothek gelegt und gleichzeitig für das Gebäude ein klares und auf die ursprüngliche Architektur bezogenes ästhetisches Konzept entwickelt. Dazu wurden tragende Gestaltungselemente der ursprünglichen Architektur wiederhergestellt: So waren in den ursprünglichen Galerieräumen unterhalb eines Stuckfrieses Messingzapfen zum Aufhängen der Bilder vorhanden, die jedoch in der Folgezeit überstrichen, überdeckt und teilweise entfernt worden waren. Im Rahmen der Renovierung wurden alle vorhandenen Zapfen wieder sichtbar gemacht; im zentralen Kuppelsaal dienen sie nun zur Befestigung großer schwarzer Platten, die einerseits als schallschluckende Elemente dienen, jedoch zugleich auch ein architektonisches Zitat oder eine Reminiszenz an die Geschichte des Gebäudes als frühere Galerie bilden. Die Ästhetik dieses zentralen Raumes wird durch einen Kristallleuchter, der anhand von Bildern und von auf dem Dachboden aufgefundenen Originalteilen rekonstruiert werden konnte, in besonderer Weise unterstrichen.

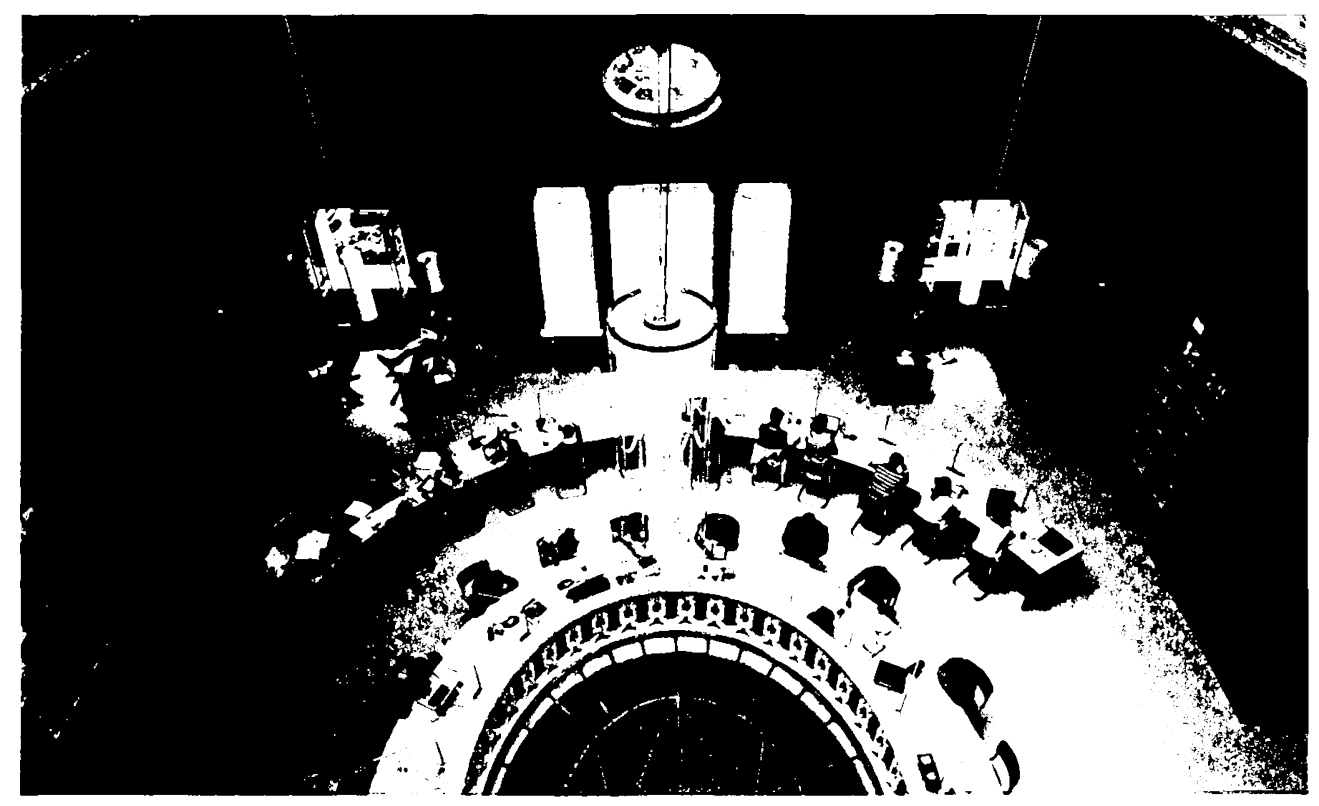

Abb. 2: In der Medizinischen Lesehalle zeigt sich nach der Renovierung die besondere JugendstilÄsthetik des ursprünglich als Galerie dienenden Gebäudes.

Foto: Sebastian Arlt 
Die gesamte Möblienung wurde unter Berücksichtigung der Ästhetik des Raumes eigen angefertigt. In den Leseräumen dominiert ein helles Linoleum, das nicht nur als Fußbodenbelag ausgelegt ist, sondern sich auch über die Flächen der Tische zieht, dort als Schreibunterlage dient und zugleich mit dem Eichenfurnier der Tische farblich harmoniert.

Mit der Wiedereröffnung der Bibliothek im Juni 2013 - fast genau 100 Jahre nach des Erbauung der heutigen Bibliothek als Galeriegebäude - präsentiert sich die Medizinische Lesehalle als ein sehr ästhetisch gestalteter, die besondere Geschichte des Gebäudes aufnehmender Ort mit sehr hoher Aufenthaltsqualität; letzteres bezeugt auch die durchwe६ hohe Auslastungsquote der Arbeitsplätze an sieben Tagen in der Woche. Zur neuen Aufenthaltsqualität gehört auch das - bei Nutzern wie Bibliotheksmitarbeitern - nicht immer unumstrittene Konzept des weitgehenden Abbaus von Zutrittsbarrieren. Für dieses Zie. wurden nicht nur die physischen Barrieren wie Drehkreuze und Schranken ersatzlos de. montiert, sondern es wurde auch bewusst auf eine Garderobenpflicht verzichtet und für die Sicherung des Bestandes ganz auf RFID-Technik vertraut. Die besondere Ästhetik dieses Gebäudes bringt eine ganz neue Nutzergruppe in die Bibliothek, die sich weniger für die Literatur und auch nicht für die Arbeitsplätze, sondern für das Gebäude an sich interessiert, nicht selten frühere Medizinstudierende, die über die Veränderung ,ihrer' alten Bibliothek staunen. Mit der Nominierung des Gebäudes für die ,Architektouren' 2014, einer von der Bayerischen Architektenkammer getroffenen Auswahl besonders gelungener Neu- und Umbauten des Vorjahres, sowie einer lobenden Erwähnung beim Fassadenpreis der Landeshauptstadt München 2015 erfuhr der Umbau der Medizinischen Lesehalle auch eine offizielle und fachliche Anerkennung.

Das aktuelle, aber keineswegs letzte Bauvorhaben der Universitätsbibliothek der LMU, das Philologicum, unterscheidet sich von den vorangegangenen Projekten nicht nur hinsichtlich der Dimension, sondern auch durch eine besonders lange und intensive Vorbereitungszeit. Abgesehen von der Medizinischen Lesehalle wird es die einzige Bibliothek sein, die ein ganzes Gebäude für sich in Anspruch nehmen kann und sich nicht mit Lehrstühlen und Hörsälen unter einem Dach befindet. Dennoch wird auch das Philologicum in unmittelbarer Nähe zu der nutzenden Fakultät und zudem an besonders exponierter Stelle liegen: Das Gebäude entsteht an der Ecke von Schelling- und Ludwigstraße und liegt damit nicht nur an der zentralen Prachtstraße Münchens zwischen Feldhermhalle und Siegestor, sondern außerdem sowohl der Bayerischen Staatsbibliothek als auch dem Hauptgebäude der LMU und damit auch der Zentralbibliothek gegenüber.

Diese Lage war eine der wesentlichen Herausforderungen für den neuen Entwurf, denn der dafür vorgesehene Platz wurde bereits durch ein besonders herausgehobenes Gebäude eingenommen. In der historischen Fassade des 1833-35 von dem Ludwigstraßen-Architekten Friedrich von Gärtner ursprünglich als Münchner Blindeninstitut errichteten Gebäudes waren - nach Beschädigung im Krieg und weitgehender Entkernung der ursprünglichen Bausubstanz - seit den 1960er Jahren die Lehrstühle der Romanistik nebst Hörsälen und der zugehörigen Institutsbibliothek untergebracht. Die erste organisatorische Aufgabe des Projekts Philologicum bestand deshalb darin, Lehrstühle und Bibliothek provisorisch unterzubringen; die viel größere, architektonische Herausforderung war jedoch, die unbedingt erhaltenswerte und unter Denkmalschutz stehende Gärtnerfassade im Osten und Norden des Gebäudes samt zweier sogenannter Eckrisalite im Westen in ein völlig neu zu planendes und modernen Anforderungen entsprechendes Bibliotheksgebäude zu integrieren, und zu- 
gleich die damit schon weitgehend vorgegebene Kubatur mit den Nutzungsanforderungen in Übereinstimmung zu bringen. Zum späteren Zeitpunkt kam für die Bauingenieure noch die schwierige Anforderung hinzu, die 19,7 Meter hohe Fassade stabil zu halten, während das gesamte übrige Gebäude nicht nur entkernt, sondern vollständig abgetragen wurde.

Bei der Planung dieses großen Bibliotheksgebäudes erwies sich der lange Vorlauf als ein großer Vorteil, auch wenn einige Parameter des Projekts im Laufe der Zeit verändert werden mussten. Bereits 2002 wurde eine erste Planungskommission für die Errichtung eines Philologicums ins Leben gerufen und am Ende des nächsten Jahres schon der Bauantrag an das Ministerium übermittelt. Von da an vergingen jedoch nochmals rund zehn Jahre, bis - bei einem Besuch des Ministerpräsidenten an der Universität im April 2013 - grünes Licht für den Bau gegeben wurde. Danach musste alles schnell gehen: Noch im gleichen Frühjahr wurde aufgrund der rasch aktualisierten Bedarfsanforderung der Universitätsbibliothek das Raumprogramm erstellt, im Spätsommer wurde der Architektenwettbewerb ausgelobt, im März des Folgejahres tagte das Preisgericht.

Bereits vor dem eigentlichen Planungsstart hatte die Universitätsbibliothek begonnen, den Bestand der elf zu integrierenden, sehr unterschiedlich großen Bibliotheken in mehreren Retro-Projekten vollständig im Online-Katalog zu erfassen. Der vollständige Katalognachweis war Voraussetzung für das Aufstellen des Mengengerüstes, bej dem auch der Anteil der Dubletten geschätzt oder ermittelt werden musste. Dabei zeigte sich, dass im Bereich der philologischen Literatur unzählige Ausgaben und Auflagen einen formalen Dublettenbegriff ad absurdum führen und daher letztlich nur eine intellektuelle Sichtung Klarheit bringen kann. Zugleich mit der Retrokonversion wurden die RVK-Notationen hinterlegt und ein Teil der Bibliotheken schon vor Beginn der Bauplanung umgestellt.

Mit der Aufstellung des Raumprogrammes musste eine Grundsatzentscheidung getroffen werden: War die ursprüngliche Bedarfsmeldung - in der Tradition der bisherigen Bibliotheksbauprojekte der LMU - sehr stark vom vorhandenen und unterzubringenden Buchbestand dominiert und damit die Zahl der vorzusehenden Arbeitsplätze letztendlich eine Residualgröße, so wurde diese Vorgehensweise nun grundlegend durch ein nutzungsorientiertes Konzept abgelöst und der Bestand aufgrund der verfügbaren Nutzfläche auf rund 420.000 Bände begrenzt. Dies erlaubte es, die Anzahl der Arbeitsplätze auf ein Minimum von 700 festzulegen. Bei rund 8.000 Studierenden in der Fakultät für Sprach- und Literaturwissenschaften als potenziellen Nutzern ein keineswegs zu hoher und immer noch unter den entsprechenden DIN-Ansätzen liegender Wert. Möglich war dies nur, indem neben einer Aussonderung von dubletter und ephemerer Literatur sowie der verstärkten Umstellung auf E-Journals auch die Magazinierung eines größeren Teiles des Bestandes geplant wurde - freilich nicht in dem Neubau selbst, denn dies wäre wiederum zu Lasten der Arbeitsplätze gegangen, sondern in einem außerhalb neu angemieteten (und später in einem neu zu errichtenden) Außenmagazin der Zentralbibliothek. Auch hier zeigte sich die gute Zusammenarbeit zwischen der Universitätsbibliothek und der LMU-Liegenschaftsverwaltung, da die Liegenschaftsverwaltung für den Gedanken, nochmals zusätzliche Magazinfläche zur Verfügung zu stellen, rasch gewonnen werden konnte.

Das quantitativ auf den beiden Grundparametern - rund 420.000 Bände als Bestandsuntergrenze und 700 Arbeitsplätze als Minimum - und qualitativ besonders auf den Anforderungen unterschiedlicher Zonierungen (leise, halblaute, laute Bereiche - Einzel- und Gruppenarbeitsplätze) beruhende Raumkonzept der Universitätsbibliothek war eine we- 
sentliche Grundlage für den Architektenwettbewerb, an dem neben dem Direktor der Universitätsbibliothek noch ein weiterer, bausachverständiger bibliothekarischer Kollege als Sachpreisrichter mitwirkte und in den die Universitätsbibliothek durch eine Mitarbeiterin auch in die Vorprüfung eingebunden war. Dass aus diesem Wettbewerb, der nach einem vorangegangenen Teilnahmewettbewerb auf 50 Teilnehmer begrenzt war, zunächst kein erster Preis hervorging, erwies sich nicht als Nachteil, sondern vielmehr als ein großer Vorteil des Verfahrens. In einer Überarbeitungsphase hatten drei jeweils ausgezeichnete Büros die Gelegenheit, mit den Nutzern in einem Dialog zu treten und ihre Entwürfe entsprechend anzupassen. Sehr eindeutig fiel anschließend die Wahl auf die Arbeitsgemeinschaft der Bregenzer Büros Fink-Thurnher/Cukrowicz-Nachbaur, deren Entwurf insbesondere durch hohe Funktionalität, niedrige Betriebskosten, vielfältige Nutzungsmöglichkeiten und die Vermittlung unterschiedlicher Raumatmosphären überzeugte ${ }^{14}$.

In einem sehr konstruktiven Dialog zwischen dem Architekturbüro, dem Staatlichen Hochbauamt München 2, dem LMU-Liegenschaftsreferat und der Universitätsbibliothek ließ sich der überarbeitete Wettbewerbsbeitrag sehr schnell in einen auszuführenden Entwurf umsetzen, wobei auch seitens der Bibliothek noch weitere Feinjustierungen vorgenommen wurden, wie zum Beispiel eine nochmalige Überarbeitung des Zonierungskonzeptes, die Einrichtung einer Kommunikationszone im Südturm und von Konzentrationszonen im Nordturm des Gebäudes sowie eines frei zugänglichen Kompaktmagazins im Untergeschoss. Von Vorteil erwies es sich, dass die Projektleitung und das Bauteam der Universitätsbibliothek von Anfang an in die Bau-Jour-fixes und auch in die FachplanerRunden einbezogen waren und alle baulichen Schritte begleiten. Eine ebenso hohe Priorität kommt im ganzen Prozess dem Dialog mit der Fakultät und den beteiligten Fächern zu. Alle Baupläne wurden den Fachvertretern ausführlich vorgestellt; der Dekan der Fakultät wirkte auch als Sachpreisrichter beim Wettbewerb mit. Bereits mit Baubeginn mussten in den elf bestehenden Bibliotheken einschneidende Veränderungen vorgenommen werden. Die Bibliothek für Romanistik hatte als Erste aus ihren angestammten Räumlichkeiten im abzureißenden Gebäude zu weichen; bei dieser Gelegenheit wurde der Bestand bereits weitgehend auf die künftige Zielgröße reduziert und vollständig auf RVK umgestellt. Die übrigen Bibliotheken werden zwar bis zum Umzug in ihren bisherigen Räumlichkeiten verbleiben, die Umstellung auf RVK und die Entnahme der für die Magazinierung bzw. für die Aussonderung vorgesehenen Bestandssegmente soll jedoch nach Möglichkeit bis zum Umzug abgeschlossen sein. Auch dies ist nur in der bestehenden engen Kooperation mit der Fakultät möglich, die für jedes Einzelfach ,Sichtungsberechtigte' benannt hat, die für die Magazinabgaben bzw. Aussonderungsentscheidungen verantwortlich zeichnen.

Mit der Fertigstellung des Philologicums wird voraussichtlich zum Jahreswechsel 2018/19 die größte Fachbibliothek an der LMU, aber auch eine der größten Freihandbibliotheken in München eröffnet und dabei sowohl das Bibliothekssystem der Universitätsbibliothek der LMU wie auch die Universität als Ganze durch seine herausgehobene Lage, seine Dimension und wegen des wohldurchdachten Nutzungskonzeptes in besonderer Weise sichtbar machen.

14 Schüller-Zwierlein, André: Architektenwettbewerb für das Philologicum ist entschieden. Die Bauplanungen für die lang erwartete neue Fachbibliothek der Universitätsbibliothek der LMU können beginnen. In: Bibliotheksforum Bayem 8 (2014), S. 313-315. 


\section{Ausblick}

Aus Sicht der Universitätsbibliothek käme mit der Eröffnung des Philologicums der Prozess der Bildung von Fachbibliotheken fast an sein natürliches Ende, da mit Ausnahme der Rechtswissenschaften und einiger Fächer im Bereich der Kulturwissenschaften inzwischen das gesamte Fachspektrum der LMU durch Fachbibliotheken abgedeckt ist. Ein großes bauliches Desiderat besteht allerdings weiterhin im Bereich der Zentralbibliothek, die bisher mehr einen Verwaltungstrakt mit angegliedertem Lesesaal und Ausleihhalle als eine heutigen Nutzungsanforderungen entsprechende Bibliothek darstellt. Dass die Lehrbuchsammlung nochmals in einem eigenen und viele Straßenblocks entfernt liegenden Gebäude untergebracht ist, gehört zu den augenfälligsten Monita. Doch während die bauliche Veränderung der Zentralbibliothek zunächst noch ein Desiderat ist, entstehen im dezentralen Bereich bereits neue, ganz konkrete Bau- und Umzugsprojekte: Im Zusammenhang mit der langfristigen Flächenplanung der LMU werden gleich eine Reihe von Fächern an einen neuen Standort ziehen, was auch eine Verlagerung der entsprechenden Fachbibliotheken mit sich bringen wird: Zunächst wird die Tiermedizin von ihrem bisherigen Standort am Englischen Garten in das nördlich von München gelegene Oberschleißheim - fast 20 Kilometer vom LMU-Hauptgebäude entfernt - ziehen. Der bisherige Standort der Tiermedizin wird neuer Sitz der Physik. Dafür wird die bisherige Fachbibliothek für Physik und Mathematik aufgelöst, und eine Physikbibliothek wird auf dem neuen Physik-Campus entstehen. Die verbleibende Mathematik zieht in das freiwerdende Gebäude der bisherigen Frauenklinik südlich des Stadtzentrums und wird dabei die bisher in zwei anderen Fachbibliotheken aufgestellten Bestände zur Statistik und Informatik mit aufnehmen. Voraussichtlich wird auch die gerade erst entstandene Geobibliothek nochmals aufgeteilt werden, wenn die Institute der Geologie in bisher von der Theoretischen Medizin belegte Gebäude umziehen werden.

Mit dem Bau dieser weiteren Bibliotheken folgt die LMU ihrem Grundsatz, die bibliothekarischen Einrichtungen stets in unmittelbarer Nähe der jeweiligen Fakultäten unterzubringen. So sehr dies aus Nutzersicht und auch für die enge Zusammenarbeit von Bibliothek und Fakultät von Vorteil ist, so ergibt sich daraus jedoch gleichzeitig eine weitere räumliche Zersplitterung des Bibliothekssystems als notwendige Konsequenz erstens des engen räumlichen Zusammenhangs von Bibliothek und Lehre und Forschung und zweitens der immer stärkeren räumlichen Ausdehnung der LMU, auch über die Stadtgrenzen von München hinaus. Für die Universitätsbibliothek entstehen neue Anforderungen, insbesondere an die Personal- und Arbeitsorganisation; erforderlich werden aber auch zusätzliche Ressourcen, um an künftig knapp 20 Standorten gleichmäßig lange Öffnungszeiten abdecken und einen einheitlichen Service anbieten zu können.

\section{Schlussbetrachtung}

Im Verlauf von drei Jahrzehnten, insbesondere jedoch in der letzten Dekade, ist es der Unjversitätsbibliothek der LMU gelungen, ein einheitliches und nun fast alle an der Universität vertretenen Fächer abdeckendes Bibliothekssystem aufzubauen. 
Neben der baulichen Veränderung ist es gelungen, einheitliche Services an allen Fachbibliotheken, angeglichene Geschäftsgänge und ein gemeinsames Selbstverständnis zu entwickel ${ }^{15}$. Eine wichtige Voraussetzung dafür war, dass - von einigen kleineren Ausnahmen abgesehen - das bibliothekarische Fachpersonal bei der Universitätsbibliothek etatisiert ist. $\mathrm{Zu}$ den noch nicht abschließend gelösten Fragen gehört dagegen das Finanzsystem, das sich aus einer Vielzahl zentraler und auf unterschiedlichen hierarchischen Ebenen angesiedelter dezentraler Mittel speist; was klare Verantwortungs- und Kompetenzregelungen nicht leicht macht.

Fast immer waren es bauliche Maßnahmen, die den Prozess der bibliothekarischen Vereinheitlichung und Neuorganisation einleiteten und das heutige System der UB der LMU prägen $^{16}$. Ohne diese baulichen Veränderungen würde das Bibliothekssystem der LMU vermutlich noch heute in dem beklagenswerten Zustand der langen Nachkriegsjahrzehnte verharren. Dass dabei die meisten Bauprojekte - das Philologicum ausgenommen - nicht als originär von bibliothekarischer Seite angestoßene Projekte, sondern im Zuge von liegenschaftlichen Notwendigkeiten und Veränderungen entstanden sind, erwies sich dabei keineswegs als Nachteil, auch wenn von manchen lehrbuchmäßigen Vorgehensweisen Abstand genommen werden musste. Die liegenschaftliche Notwendigkeit erweist sich zumindest an der LMU als der stärkste Motor für Veränderungen, stärker in jedem Falle als der bloße bibliothekarische Appell und Verweis auf Wünsche und Bedarfe.

15 Schüller-Zwierlein, André: Der Ort der Dienstleistung. In: Handbuch Hochschulbibliothekssysteme. Leistungsfähige Informationsinfrastrukturen für Wissenschaft und Studium, hrsg. von Konstanze Söllner und Wilfried Sühl-Strohmenger. Berlin [u. a.] 2014, S. 241-248.

16 Die Auswirkungen von Bibliotheksbauten auf die Entwicklung zweischichtiger Bibliothekssysteme haben Söllner und Sühl-Strohmenger in einer größer angelegten, bibliotheksvergleichenden Studie untersucht. Siehe dazu: Söllner, Konstanze; Sühl-Strohmenger, Wilfried: Planen und Bauen von zukunftsfähigen Hochschulbibliothekssystemen - zentral und dezentral. In: ABI-Technik 35 (2015), S. 82-94. 